\title{
Transient anomalous diffusion of tracer particles in soft matter
}

\author{
Scott A. McKinley \\ Department of Mathematics, Duke University, P.O. Box 90320, Durham, \\ North Carolina 27708 \\ Lingxing Yao \\ Department of Mathematics, University of Utah, 155 South 1400 East Room 233, \\ Salt Lake City, Utah 84112

\section{Gregory Forest ${ }^{\text {a) }}$} \\ Department of Mathematics and Department of Biomedical Engineering, \\ Institute for Advanced Materials, University of North Carolina at Chapel Hill, \\ Chapel Hill, North Carolina 27599-3250
}

(Received 30 April 2009; final revision received 8 September 2009)

\begin{abstract}
Synopsis
This paper is motivated by experiments in which time series of tracer particles in viscoelastic liquids are recorded using advanced microscopy. The experiments seek to infer either viscoelastic properties of the sample [Mason and Weitz, Phys. Rev. Lett. 74, 1250-1253 (1995)] or diffusive properties of the specific tracer particle in the host medium [Suh et al., Adv. Drug Delivery Rev. 57, 63-78 (2005); Matsui et al., Proc. Natl. Acad. Sci. U.S.A. 103, 18131-18136 (2006); Lai et al., Proc. Natl. Acad. Sci. U.S.A. 104, 1482-1487 (2007); Fricks et al., SIAM J. Appl. Math. 69, 1277-1308 (2009)]. Our focus is the latter. Experimentalists often fit data to transient anomalous diffusion: a sub-diffusive power law scaling $\left(t^{\nu}\right.$, with $\left.0<\nu<1\right)$ of mean-squared displacement (MSD) over a finite time interval, with longtime viscous scaling $\left(t^{1}\right)$. The time scales of sub-diffusion and exponents $\nu$ are observed to vary with particle size, particle surface chemistry, and viscoelastic properties of the host material. Until now, explicit models for transient subdiffusive MSD scaling behavior [Doi and Edwards, The Theory of Polymer Physics (Oxford University Press, New York, 1986); Kremer and Grest, J. Chem. Phys. 92, 5057-5086 (1990); Rubinstein and Colby, Polymer Physics (Oxford University Press, New York, 2003)] are limited to precisely three exponents: monomer diffusion in Rouse chain melts $\left(t^{1 / 2}\right)$, in Zimm chain solutions $\left(t^{2 / 3}\right)$, and in reptating chains $\left(t^{1 / 4}\right)$. In this paper, we present an explicit parametrized family of stochastic processes (generalized Langevin equations with prescribed memory kernels) and derive their closed-form solutions which (1) span the complete range of transient sub-diffusive behavior and (2) possess the flexibility to tune both the time window of sub-diffusive scaling and the power law exponent $\nu$. These results establish a robust family of sub-diffusive models, for which the inverse problem of parameter inference from experimental data [Fricks et al., SIAM J. Appl. Math.
\end{abstract}

a) Author to whom correspondence should be addressed; electronic mail: forest@amath.unc.edu 
69, 1277-1308 (2009)] remains to be developed. () 2009 The Society of Rheology. [DOI: 10.1122/1.3238546]

\section{DIFFUSION IN A VISCOELASTIC MEDIUM}

\section{A. The generalized Langevin equation}

Over the past half-century, the molecular theory of polymer dynamics [Rouse (1953); Zimm (1956); Kubo (1985); Kremer and Grest (1990); Levine and Lubensky (2001)] has developed the correspondence between diffusive dynamics and linear viscoelastic relaxation of polymer melts and solutions. Excellent accounts of the sources and scaling properties of diffusive time scales and associated normal modes of stress relaxation are given in the monographs of Doi and Edwards (1986) and Rubinstein and Colby (2003). Stochastic models were likewise developed to understand atomic and molecular fluctuation spectra in media where viscous and elastic collisions are comparable [Mori (1965); Zwanzig and Bixon (1970)].

A standard model for diffusion in a viscoelastic medium is a generalized Langevin equation (GLE). Following Mori (1965), Zwanzig and Bixon (1970), and Mason and Weitz (1995), the Stokes drag law is generalized by convolution of the velocity with a memory function. The path $X(t)$ of a tracer particle of mass $m$ is modeled by

$$
m \ddot{X}(t)=-\int_{0}^{t} \Gamma(t-s) \dot{X}(s) d s+F(t),
$$

where $F(t)$ is a Gaussian colored noise with autocorrelation related to the memory kernel by the fluctuation-dissipation theorem (with $k_{B}$ as the Boltzmann constant and $T$ as absolute temperature),

$$
\langle F(t) F(s)\rangle=k_{B} T \Gamma(|t-s|) .
$$

For simplicity, we study the dynamics in one dimension. In Laplace transform space, the memory drag law regains the multiplicative form of the simple Langevin equation for viscous diffusion, except with a frequency-dependent drag coefficient given by the transform of the memory kernel. The GLE in transform space becomes

$$
m z^{2} \tilde{X}(z)=-\widetilde{\Gamma}(z) z \tilde{X}(z)+\widetilde{F}(z) .
$$

In the viscous limit, $\widetilde{\Gamma}$ reduces to the constant Stokes drag coefficient $\beta=6 \pi a \eta$, where $\eta$ is the fluid viscosity and $a$ is the particle radius.

GLE models have received significant attention in the past 15 years in the field of passive microrheology [Mason and Weitz (1995)], where tracer particles are embedded in viscoelastic media and tracked using modern microscopy techniques. The goal of passive microrheology, consistent with the history of polymer molecular theory, is to infer frequency-dependent linear viscoelastic moduli from particle fluctuations in frequency space, assuming the diffusive-stress relaxation correspondence of polymers melts and solutions applies to diffusing probe particles. Outside the scope of this paper lies another body of literature on active microrheology where a deterministic forcing is applied directly to the particle (e.g., magnetic beads) and observations focus on either the particle motion or the fluid response.

Our interest in GLEs, motivated by experiments for the Virtual Lung Project at the University of North Carolina (UNC) in the Superfine group, in the DeSimone Laboratory at UNC, and Hanes Laboratory at Johns Hopkins, and following the theoretical approach of Fricks et al. (2009), is to model diffusion in viscoelastic media for applications in 
biology and medicine. To do so, we seek stochastic models that can be applied to experimental data of tracked particles; applications of immediate interest are for diffusive transport through pulmonary mucosal layers [Matsui et al. (2006); Suh et al. (2005); Lai et al. (2007); Gratton et al. (2008)]. A standard practice in the microrheology and drug delivery literature is to plot mean-squared displacement (MSD) of particle position data on a log-log plot, from which one routinely observes a sub-diffusive MSD scaling $\left(t^{\nu}, 0<\nu<1\right)$ over an intermediate dynamic range. When the data are taken for a long time, one observes a passage to diffusive scaling $\left(t^{1}\right)$ [Suh et al. (2005)]. Consistent with various authors [Rubinstein and Colby (2003)], we refer to this feature as transient anomalous diffusion. If one varies the particle diameter with controlled surface chemistry, as in Lai et al. (2009) and Suk et al. (2009), then the observed MSD scaling varies significantly, especially as the particle diameter is nearby dominant pore/mesh scales in the polymeric network. If one controls the particle diameter and modifies surface chemistry, then physics of adhesion or repulsion of nearby polymer chains can likewise dramatically alter the diffusive paths. For the present paper, the models are blind to these detailed physics, although we continue to explore the explicit connection between particle size, medium microstructure, and particle-medium chemical interactions.

For direct modeling of tracer particles in a viscoelastic medium, a sufficiently robust family of stochastic processes is needed that reflects these fundamental MSD signatures of transient sub-diffusion. This is the primary result of the present paper. With a robust family of stochastic processes in hand, then one can build inference methods to fit model parameters to experimental data and give a means to characterize diffusive properties of a given particle in a host medium. The development of inference methods is in progress, but not completed. Such tools can be used in medicine, for example, to explore passage times through mucosal barriers of different environmental particles or drug carrier particles as functions of their surface chemistry and size distribution. We emphasize that our planned applications to biology and medicine require tools beyond characterization of diffusive or viscoelastic properties. With numerically efficient and tractable direct (time domain) models of viscoelastic diffusion, one gains the ability to simulate application scenarios, such as first passage time distributions and MSD statistics. With exactly solvable models, one gains the potential to extract scaling behavior of diffusive transport statistics with respect to model parameters, circumventing the need to perform massive parameter simulations. The results presented here provide the foundational tools for these applications.

In Fricks et al. (2009), the authors explore GLEs with memory functions for the two most celebrated models of polymer chain dynamics due to Rouse and Zimm. These models exhibit transient sub-diffusive behavior with intermediate time scale MSD scaling of $t^{1 / 2}$ and $t^{2 / 3}$, respectively. The intermediate time scale is between the shortest and longest relaxation times associated with their particular diffusive spectrum [Doi and Edwards (1986); Rubinstein and Colby (2003)]. The Rouse and Zimm models assume dilute polymer chain melts and solutions, respectively; the models become increasingly complex with respect to diffusive and stress relaxation dynamics as the concentration of chains, quality of solvent for Zimm models, and chain entanglements are considered. These considerations aim to directly relate internal diffusive dynamics with stress relaxation of a viscoelastic medium, whereas we are concerned with foreign particles diffusing in such a medium. These two problems are related but it is an extremely challenging problem to infer one process from the other and we do not attempt to make that connection either in Fricks et al. (2009) or the present paper. However, we look to the polymer dynamics literature for candidate models consistent with experimental data. The Rouse and Zimm models provide strong guidance; they exhibit transient anomalous diffusion of 
a monomer with a sub-diffusive MSD power law exponent on intermediate time scales. Their main drawback is they produce exactly two exponents, $\frac{1}{2}$ and $\frac{2}{3}$, whereas we seek to capture the full range of sub-diffusive power law exponents that are reported across the literature. If we look to polymer theory for diffusive scaling behavior of entangled polymers to achieve wider ranges of MSD scaling, one finds evidence for transitions across multiple time scale windows with three distinct power law exponents [Kremer and Grest (1990); Rubinstein (personal communication)]. The major limiting factor is the discrete, sparse set of fractional exponents. Additionally, except for the Rouse and Zimm models, these results are not based on exact analysis or numerical simulations of the corresponding GLEs and the molecular dynamics simulation approaches as in Kremer and Grest (1990) are computationally prohibitive. Thus, we are led back to the Rouse and Zimm kernels to seek generalizations of the corresponding GLEs that retain a single intermediate sub-diffusive power law scaling but with the capacity to fit an arbitrary power law exponent.

In this paper, we present several advances toward this goal, which we summarize prior to presentation of the details of our techniques and analysis. The reader who is interested exclusively in the applications of these methods can focus primarily on the next section.

\section{B. A special family of GLE kernels and summary of results}

In this paper, we introduce GLEs with a family of kernels inspired by the scaling properties of the diffusive spectrum of the Rouse and Zimm kernels (Sec. II A). In previous work [Fricks et al. (2009)], we demonstrated that GLEs with Prony (exponential) series kernels and a uniform weight distribution for terms in the series can be transformed to an order $O(N)$-dimensional vector Langevin equation. After a linear algebra problem is solved, this observation leads to two advances: (1) fast and statistically accurate numerical methods for generating particle paths and MSD profiles if a Prony series kernel is specified and (2) maximum likelihood inference in which the diffusive spectra $\alpha_{k}, k=1, \ldots, N$ (rate constants in each exponential) are recovered from noisy position time series of tracer particles. The first capability is not limited by the number $N$ of discrete time scales of memory in the diffusive process or, equivalently, the number of terms in the Prony series. Results from Fricks et al. (2009) are recalled in Sec. II B for $N=4$ and a Rouse memory spectrum, showing a transient anomalous MSD scaling with $t^{1 / 2}$. We will present simulation results below for $N$ up to 20000 . The second capability, that of inference of the discrete memory spectrum from noisy position time series data, is limited in $N$. In Fricks et al. (2009), the time scales of memory in the data are sequentially captured from shortest to longest and the variance of the maximum likelihood estimator grows with each successive (larger) memory time scale. The maximum likelihood estimator illustration in Fricks et al. (2009) is with an $N=4$ Rouse spectrum, beginning from numerically generated position time series data. However, viscoelastic diffusion processes are expected to require $N \gg 1$, perhaps on the order of hundreds or thousands, for which our previous maximum likelihood methods are impractical. Just as importantly, these "time domain" methods suffer the significant limitation that exists for all polymer diffusion models. Namely, there are no available models that exhibit a MSD power law on an intermediate time window, $\left\langle x^{2}(t)\right\rangle \sim t^{\nu}$ for $t_{\min }<t<t_{\max }$, except for $\nu$ $=1 / 2$ (Rouse), 2/3 (Zimm), and 1/4 (reptation).

The models introduced in the present paper and our analysis of them overcome the limitation on $N$ by a natural three-parameter generalization of the Rouse time scale spectrum and provide explicit solutions of the corresponding GLE that fill the entire 
range of sub-diffusive transient MSD scaling. We further establish the connection between scaling properties of the memory spectrum and statistical properties of path data such as MSD.

Caveat: For these results to have an impact on the analysis of experimental data, we must solve the inference problem: given noisy position time series of tracer particles in a viscoelastic medium, infer the parameters in our model. We anticipate similar methods to Fricks et al. (2009) will apply, namely, maximum likelihood estimation of parameters and their variance, but progress on this problem is not sufficient to include in the present paper. In any case, with the present results, we bypass the limitation on the number of exponential modes and sequential recovery of the diffusive time scales from data, in favor of recovery of a three-parameter generating function for the memory kernel. Furthermore, if the data exhibit a transient MSD sub-diffusive power law, we know the present model can capture any exponent.

One additional open problem remains: to connect the physics of surface chemistry between the probe particle and the medium to the parametrized memory kernel in the GLE. We have no progress in this direction, so that we are not able at present to learn about details of particle-polymer interaction potentials.

The major analytical results begin in Sec. II C where we establish a remarkable "exact solvability" result for the weak zero mass limit. The standard representation of particle position in Laplace or frequency space [Mason and Weitz (1995)] can be explicitly inverted back into the time domain. This result holds for any choice of the discrete time scale spectrum, but the analysis exploits a uniform weight distribution for terms in the Prony series. Thus, we deduce an explicit particle path representation in the time domain or, equivalently, an exact solution $X(t)$ of the integro-differential GLE equation for zero mass, which is of the form

$$
X(t)=C B(t)+Z(t),
$$

where $B(t)$ is a standard Brownian motion and $Z(t)$ is a stationary process whose full set of parameters can be expressed explicitly in terms of the parameters of the memory kernel $\Gamma$ [see Eqs. (14), (15), and (18)]. The stationary process $Z(t)$ is a sum of OrnsteinUhlenbeck (OU) processes, where each term represents one color of noise in the process and the full colored noise spectrum is related by explicit polynomial interpolation to the kernel memory spectrum. This result, however, is too general to analyze and/or deduce detailed statistical properties of the process, such as MSD scaling behavior. Subsequently in Sec. II D, we establish a correspondence principle between an explicit scaling behavior of the memory spectrum and transient MSD power law behavior. For a three-parameter memory spectrum in the Prony series kernel, we analytically determine transient anomalous diffusion of the zero mass GLE process, with the ability to tune any MSD subdiffusive power law and the window of anomalous diffusion. Since we also have direct numerical tools, we illustrate several numerical MSD profiles.

The above results are achieved for uniform weights in the Prony series, a property shared by Rouse and Zimm kernels. Thus our results and their application to physical viscoelastic diffusion processes might appear to be unreasonably restrictive. Nonetheless we establish in Sec. II D that the MSD profile is robust with respect to nonuniform weight structures as long as the weights are sufficiently independent and random. This fact from probability theory has significant impact: even though the explicit calculation to invert the Laplace transform for position back into the time domain relies heavily on uniform weights, the result is robust to random perturbations of the weights.

The upshot of this sequence of results is twofold. With respect to direct modeling, we have a family of exact zero mass GLE solutions that exhibit transient anomalous diffu- 
sion with an arbitrary MSD power law exponent on intermediate time scales. And, with respect to future methods of statistical inference, the memory kernel depends on just three parameters: the shortest time scale $\tau_{1}$ of memory in the data and two additional parameters, $N$ and $\rho$, which dictate the largest memory time scale $\tau_{N}$ and the scaling behavior of the memory spectrum. Finally, the memory scaling parameter $\rho$ dictates the MSD power law exponent $\nu$.

The remaining issue surrounds the order of approximation of the zero mass limit solution with respect to a finite, nonzero mass process. As seen in Fig. 3, there is a difference in MSD between finite and zero mass, which is a consequence of the singular nature of the zero mass limit. We represent the exact solution of the full GLE with nonzero mass, $X_{m}(t)$, as a singular perturbation of the weak zero mass limit, $X(t)$,

$$
X_{m}(t)=X(t)+Y(t)+o(\sqrt{m}),
$$

where the correction term $Y(t)$ is a highly oscillatory stationary Gaussian process. The remainder goes to zero in MSD with $m$.

The asymptotic behavior of $Y(t)$ must be considered in two senses: as $m \rightarrow 0$ and as $N \rightarrow \infty$. The result is stated in Sec II E while the derivation is detailed in Appendix A, Sec. A 2. In summary, for fixed $N$, the oscillatory term $Y(t)$ goes to zero with $m$ in a weak sense, however it does not converge to zero in MSD. Rather, it gives a finite correction due to the singular nature of the zero mass limit. Looking ahead to complex biological materials for which the diffusive memory spectrum is expected to require $N \gg 1$, we present another result: for a fixed finite nonzero mass $m>0$, the MSD contribution from $Y(t)$ tends to zero with $1 / N$. This is to say the massive correction to the MSD due to mass vanished in the limit of infinitely many arbitrarily long memory time scales.

These results each have potentially important implications for the biomedical applications we have discussed. Inertial effects due to tracer mass do not contribute to the power law exponent of MSD profiles, which is dictated solely by the memory spectrum. However, in applications where the number of distinct relaxation times is small, features such as first passage times through barrier layers are measurably affected by the singular mass correction. With this summary of results in hand, we now proceed to their derivation.

\section{TRANSIENT ANOMALOUS DIFFUSION}

\section{A. GLE with Prony series memory kernel}

The general form we propose for the memory kernel is a Prony series,

$$
\Gamma(t)=\beta \sum_{k=1}^{N} e^{-\alpha_{k} t},
$$

where the parameters have known physical contact if we specialize to the dynamics of a Rouse chain melt or Zimm chain solution. This series assumes a uniform weight $\beta$ (physical units of length times viscosity, as in the Stokes drag coefficient $6 \pi a \eta$, where $a$ is particle radius and $\eta$ is viscosity). The rates $\left\{\alpha_{k}\right\}$ comprise the discrete diffusive spectrum. We routinely interchange the specification of the diffusive spectrum in terms of $\left\{\alpha_{k}\right\}$ or their reciprocals $\left\{\tau_{k}\right\}$, the diffusive memory time scales.

Up to several constants which are not relevant to our purpose here, the Rouse spectrum essentially obeys the scaling behavior [Doi and Edwards (1986)], 


$$
\alpha_{k, N} \sim \sin ^{2}\left(\frac{k \pi}{2 N}\right)
$$

and the weights of each corresponding exponential in the Prony series are uniform. The power law exponent of the resulting transient anomalous diffusion of the MSD is determined by the scaling behavior of the function $\sin ^{2}(x)$ for $x \sim 0$. Therefore, a natural generalization of the Rouse spectrum is, for a given $\rho>1$,

$$
\tau_{k}^{-1}=\alpha_{k} \sim\left(\frac{k}{N}\right)^{\rho} \tau_{1}^{-1}
$$

where $\tau_{1}$ is the shortest memory time scale of the stochastic process. Such a spectrum samples from the function $x^{\rho}$ in an analogous manner to the way the Rouse spectrum samples from $x^{2}$. This scaling behavior has precedent in the polymer dynamics literature when the Zimm model for dilute polymer chains is manipulated to reflect the fractal radius of a chain with $N$ monomers. In this case, the parameter $\rho$ is a reflection of the quality of the solvent [Rubinstein and Colby (2003)].

The form of diffusive spectrum $\alpha_{k} \sim(k / N)^{\rho} \tau_{1}^{-1}$ yields a Prony series (6) that approximates a power law for all $t>\tau_{1}$. Indeed, a quick calculation reveals that

$$
\Gamma(t):=\frac{1}{N} \sum_{k=1}^{N} e^{-(k / N)^{\rho} t / \tau_{1}} \sim t^{-1 / \rho}
$$

for $t>\tau_{1}$. In this way, the GLE with Prony series kernel can be seen as an approximation of power law decay models employed in Morgado et al. (2002), Kou and Xie (2004), Vainstein et al. (2008), and Santamaria-Holek (2008). In these references, the so-called Hurst parameter $H \in(1 / 2,1)$ determines the exponent of uniform anomalous diffusion. Our parameter $\rho$ corresponds to $H$ by the relation $\rho=(3-2 H)^{-1}$, although we allow for arbitrarily large values for $\rho$ and our processes are anomalous on finite time scales.

\section{B. Transient anomalous diffusion}

A number of authors have discussed anomalous diffusion on asymptotically long time scales. In particular, Morgado et al. (2002), Santamaria-Holek (2008), and Vainstein et al. (2008) noted the fundamental relationship between the near zero behavior of the memory kernel $\Gamma$ in frequency space and the large time scaling of the MSD of the GLE. Namely, if $\widetilde{\Gamma}$ satisfies

$$
\lim _{z \rightarrow 0} \frac{\widetilde{\Gamma}(z)}{z^{\alpha}}=C_{0}
$$

then for $\nu=1+\alpha$,

$$
\lim _{t \rightarrow \infty} \frac{1}{t^{\nu}}\left\langle X^{2}(t)\right\rangle=C_{1},
$$

for some nonzero constants $C_{0}$ and $C_{1}$. When $\nu=1$, the process is called diffusive. For $\nu<1$, it is called sub-diffusive.

The Laplace transform of an exponential series is given by 


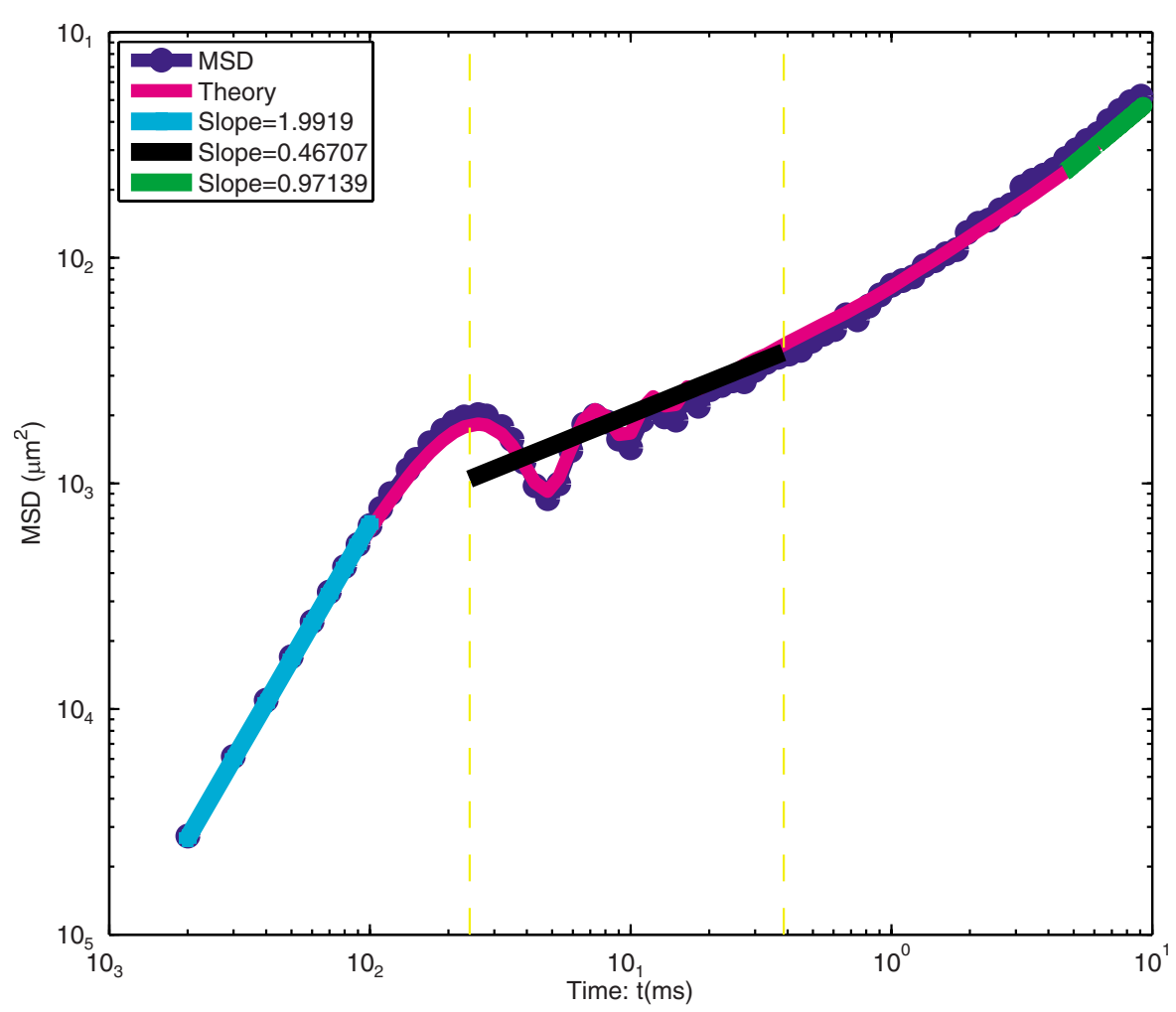

FIG. 1. MSD for a GLE with four relaxation modes and a Rouse spectrum. Two hundred paths are generated for a massive $1 \mu \mathrm{m}$ diameter bead [see Fricks et al. (2009) for further simulation details]. The MSD profile of the simulation closely reflects the predicted $2-\frac{1}{2}-1$ transition in the MSD power law from early to intermediate to large time scales, Eq. (13). See also, Fig. 3.6 in Fricks et al. (2009).

$$
\widetilde{\Gamma}(z)=\beta \sum_{k=1}^{N} \frac{1}{z+\alpha_{k}},
$$

so that the near-zero exponent $\alpha$ from Eq. (10) is zero. Therefore in the Prony series GLE setting, the large time scale dynamics are diffusive. However, over intermediate time scales (possibly longer than any given experimental time scale), the Prony series GLE exhibits sub-diffusive behavior.

As seen in the Fig. 1 from Fricks et al. (2009), the Rouse spectrum yields

$$
\left\langle X^{2}(t)\right\rangle \sim t^{\nu}, \quad \text { with } \nu=\left\{\begin{array}{cc}
2 & t \leq \tau_{1} \\
1 / 2 & \tau_{1} \leq t \leq \tau_{N} \\
1 & t \geq \tau_{N}
\end{array}\right.
$$

where $\tau_{1}$ and $\tau_{N}$ are the shortest and longest time scales, respectively. The exponents $\frac{1}{2}$ and 1 match observations for Rouse monomers [Doi and Edwards (1986); Kremer and Grest (1990)] on the intermediate and large time scales. There is a discrepancy on the shortest time scale because the present model has nonzero mass, whereas Doi and Edwards (1986) and Kremer and Grest (1990) neglect the inertial term. 


\section{GLE in the weak zero mass limit}

The key analytical advance arises from our observation that by taking the mass to zero in frequency space, the rational function for the Laplace transform of position can be explicitly inverted back to the time domain. To our knowledge, this is a completely new result that generalizes the analogous limit of viscous diffusion: a Brownian motion with a renormalized diffusion constant suffices as a proxy for viscous diffusion.

We defer the details of the derivation of the weak zero mass limit to Appendix A. The outcome is the explicit path representation of GLE solutions with zero mass,

$$
X(t)=C_{0} B_{0}(t)+\sum_{j=1}^{N-1} C_{j} Z_{j}(t),
$$

where $B_{0}$ is a standard Brownian motion and each $Z_{j}$ is an OU process associated with a specific relaxation time of the system. The coefficients are

$$
\begin{gathered}
C_{0}=\sqrt{\frac{2 k_{B} T}{\beta N \bar{\tau}}}, \\
C_{j}=\frac{p\left(-r_{j}\right)}{r_{j} p^{\prime \prime}\left(-r_{j}\right)}\left(\sum_{k=1}^{N} \frac{\alpha_{k}}{\left(\alpha_{k}-r_{j}\right)^{2}}\right)^{1 / 2},
\end{gathered}
$$

and the OU processes satisfy

$$
d Z_{j}(t)=-r_{j} Z_{j}(t) d t+\sqrt{2 k_{B} T} d B_{j}(t),
$$

where the family $\left\{B_{j}(t)\right\}, j=0, \ldots, N-1$ is collection of independent standard Brownian motions. The constants $\bar{\alpha}$ and $\bar{\tau}$ are given by

$$
\bar{\alpha}=\frac{1}{N} \sum_{k=1}^{N} \alpha_{k}, \quad \bar{\tau}=\frac{1}{N} \sum_{k=1}^{N} \alpha_{k}^{-1},
$$

the function $p$ is given by

$$
p(x)=\prod_{k=1}^{N}\left(x+\alpha_{k}\right),
$$

and $\left\{-r_{j}\right\}_{j=1}^{N-1}$ are the roots of the polynomial $p^{\prime}(x)$. Note that because each of the roots of $p(x)$ is negative, and since $p(x)$ is continuous, the roots of $p^{\prime}(x)$ are real and negative as well.

\section{General model for transient anomalous diffusion}

The specifics of the constants in the solution (14) are a bit obtuse. One might be particularly concerned about finding the roots of a polynomial of degree on the order of $10^{5}$. However, the general form discovered by taking the zero mass limit of the GLE (1) is surprisingly robust with respect to changes in the coefficients; the roots are related to the memory spectrum by a simple interpolation theorem.

To this end, define 


$$
x_{N}(t):=\frac{\sigma}{\sqrt{N \bar{\tau}}} B_{0}(t)+\frac{1}{\sqrt{N}} \sum_{k=1}^{N} c_{k} z_{k}(t),
$$

where the $\left\{z_{k}\right\}$ are OU processes with a given spectrum $\left\{\alpha_{k}\right\}_{k=1}^{N}$ and the weights $c_{k}$ are either uniform or essentially random. We have adopted lowercase letters to indicate that the coefficients and exponents are not those of the zero mass GLE process. The form of these exact solutions has the capacity to generalize the range of MSD anomalous diffusive behavior from the two discrete power laws of Rouse and Zimm to a continuous power law range. In the presence of a generalized Rouse spectrum (8), we have the MSD profile

$$
\left\langle x_{N}^{2}(t)\right\rangle \sim t^{\nu}, \quad \text { with } \nu=\left\{\begin{array}{cc}
1 & t \leq \tau_{1} \\
1-\frac{1}{\rho} & \tau_{1} \leq t \leq \tau_{N} \\
1 & t \geq \tau_{1} .
\end{array}\right.
$$

As $\rho$ varies over $(1, \infty)$, the resulting intermediate power law attains all values in the interval $(0,1)$.

We establish the above result by assuming uniform coefficients and generalizing a classical calculation that can be found in Doi and Edwards (1986). Subsequently, we apply results from probability theory to establish that, as long as the weights are roughly of similar magnitude and are sufficiently random, the fundamental exponent of the anomalous scaling, $\nu=1-1 / \rho$, remains the same.

Taking the angle brackets to be an average over the sample space of all Brownian motions driving $x_{N}(t)$, we have that the MSD curve satisfies

$$
\left\langle x_{N}(t)^{2}\right\rangle=\frac{\sigma}{N \bar{\tau}} t+\frac{1}{N} \sum_{k=1}^{N} \frac{1}{\alpha_{k}}\left(1-e^{-\alpha_{k} t}\right)=\frac{\sigma}{N \bar{\tau}} t+\tau_{1} \sum_{k=1}^{N}\left(\frac{N}{k}\right)^{\rho}\left(1-e^{-(k / N)^{\rho} t / \tau_{1}}\right) \frac{1}{N}
$$

For small values of $t$, each term of the summand is approximately $t /\left(\tau_{1} N\right)$, which yields $\left\langle x_{N}^{2}(t)\right\rangle \sim t$. For very large values of $t$, each of the exponential terms is nearly zero and the sum is no longer time dependent. In this regime, the growth of the MSD of the zero mass limit (14) of the GLE will be dominated by the Brownian motion term.

In order to assess the intermediate sub-diffusive exponent, we recognize that the sum on the right-hand side, denoted below as $\Sigma_{N}$, is a Riemann approximation to an integral. For sufficiently large $N$,

$$
\Sigma_{N}(t)=\tau_{1} \int_{0}^{1} \frac{1}{x^{\rho}}\left(1-e^{-x^{\rho} t / \tau_{1}}\right) d x+o\left(\frac{1}{N}\right)
$$

With the substitution $y=x\left(t / \tau_{1}\right)^{1 / \rho}$, we have

$$
\Sigma_{N}(t)=t^{1-1 / \rho} \int_{0}^{\left(t / \tau_{1}\right)^{1 / \rho}} \frac{1}{y^{\rho}}\left(1-e^{-y^{\rho}}\right) d y+o\left(\frac{1}{N}\right)
$$

Define $C_{\rho}:=\int_{0}^{\infty} 1 / y^{\rho}\left(1-e^{-y^{\rho}}\right) d y$ and for each $\rho$, there is a $T_{\rho}$ such that the tail of this integral is less than any given small value $\epsilon>0$. It follows that for all $t$ roughly between $T_{\rho}$ and $N^{\rho} \tau_{1}$ : 


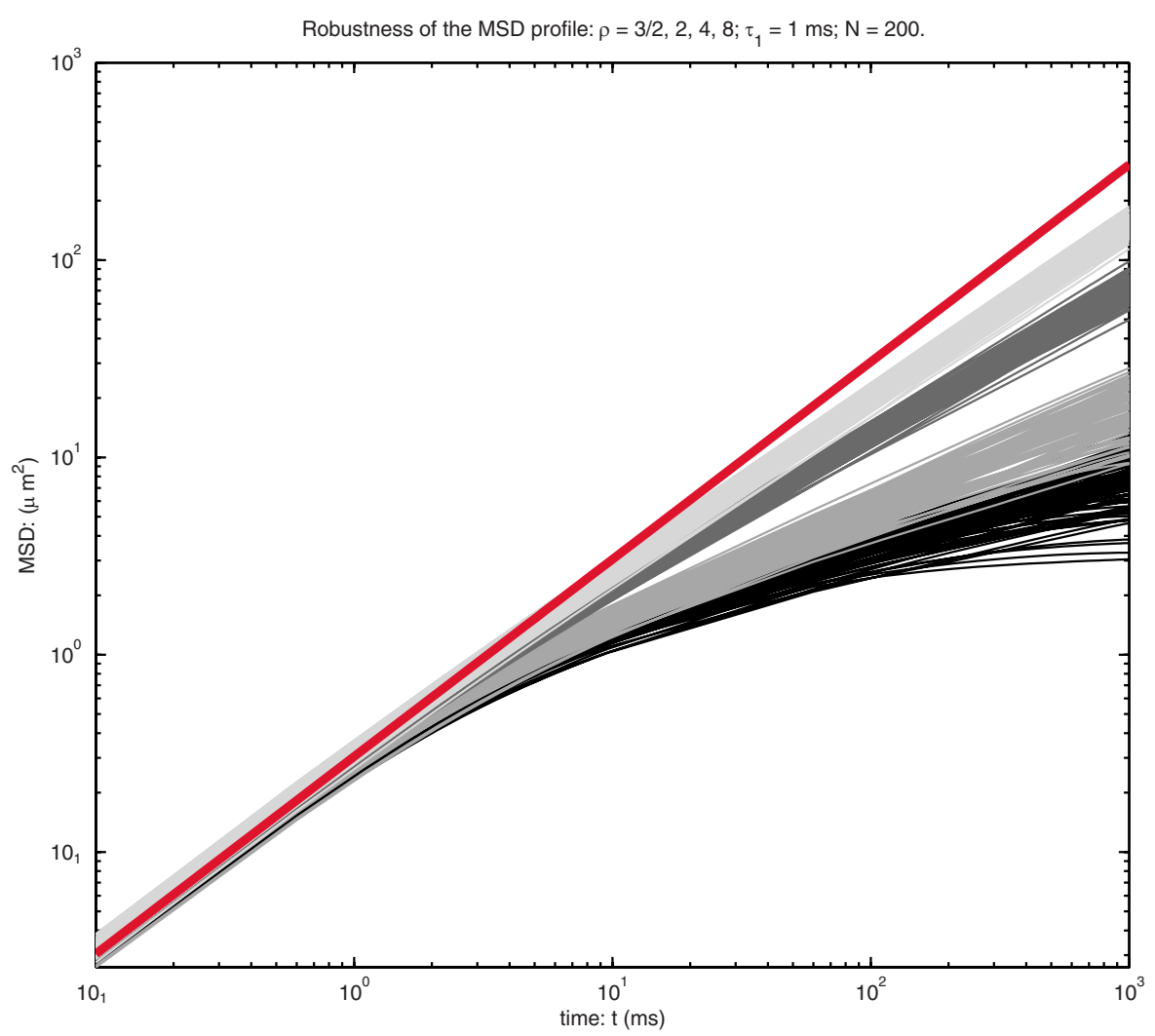

FIG. 2. We plot the MSD of the zero mass process for four different values of $\rho, \rho$ $=\{3 / 2$ (black), 2 (gray), 4 (dark gray), 8 (light gray) $\}$. In each case, the number of modes is $N=200$ and the smallest relaxation time is $\tau_{1}=1 \mathrm{~ms}$. Each line is the plot of the MSD for an independent choice of 200 random coefficients. We see that while there is variation, each value of $\rho$ has a characteristic shape. A plot of a line with slope 1 is provided for reference.

$$
\left|\Sigma_{N}(t)-C_{\rho} t^{1-1 / \rho}\right| \leq \epsilon+o\left(\frac{1}{N}\right) .
$$

It remains to show that the contribution to the MSD of the Brownian term is negligible relative to the OU terms on intermediate time scales. The coefficient, $\sigma /(N \bar{\tau})$, depends on $\rho$ in that

$$
\bar{\tau}=\frac{\tau_{1}}{N} \sum_{k=1}^{N}\left(\frac{N}{k}\right)^{\rho} \approx \tau_{1} \zeta(\rho) N^{\rho-1},
$$

where $\zeta(\rho)$ is the Riemann zeta function. Therefore, for all $t<N^{\rho-1}$, the contribution of the Brownian term is subsumed by the $o(1 / N)$ error of the Riemann approximation and can be disregarded in assessing the intermediate time scale exponent.

We defer to Appendix B the probabilistic argument for the robustness of the exponent with respect to random perturbations of the coefficients. But in Fig. 2, we graphically demonstrate the robustness of the MSD profile. For each of four values for the spectral parameter $\rho$, we randomly generate coefficients for a process with 200 modes and plot the corresponding MSD curve. One sees that each value of $\rho$ yields a characteristic shape on a $\log -\log$ plot. The argument assumes that the coefficients $c_{k, N}$ are mutually independent 


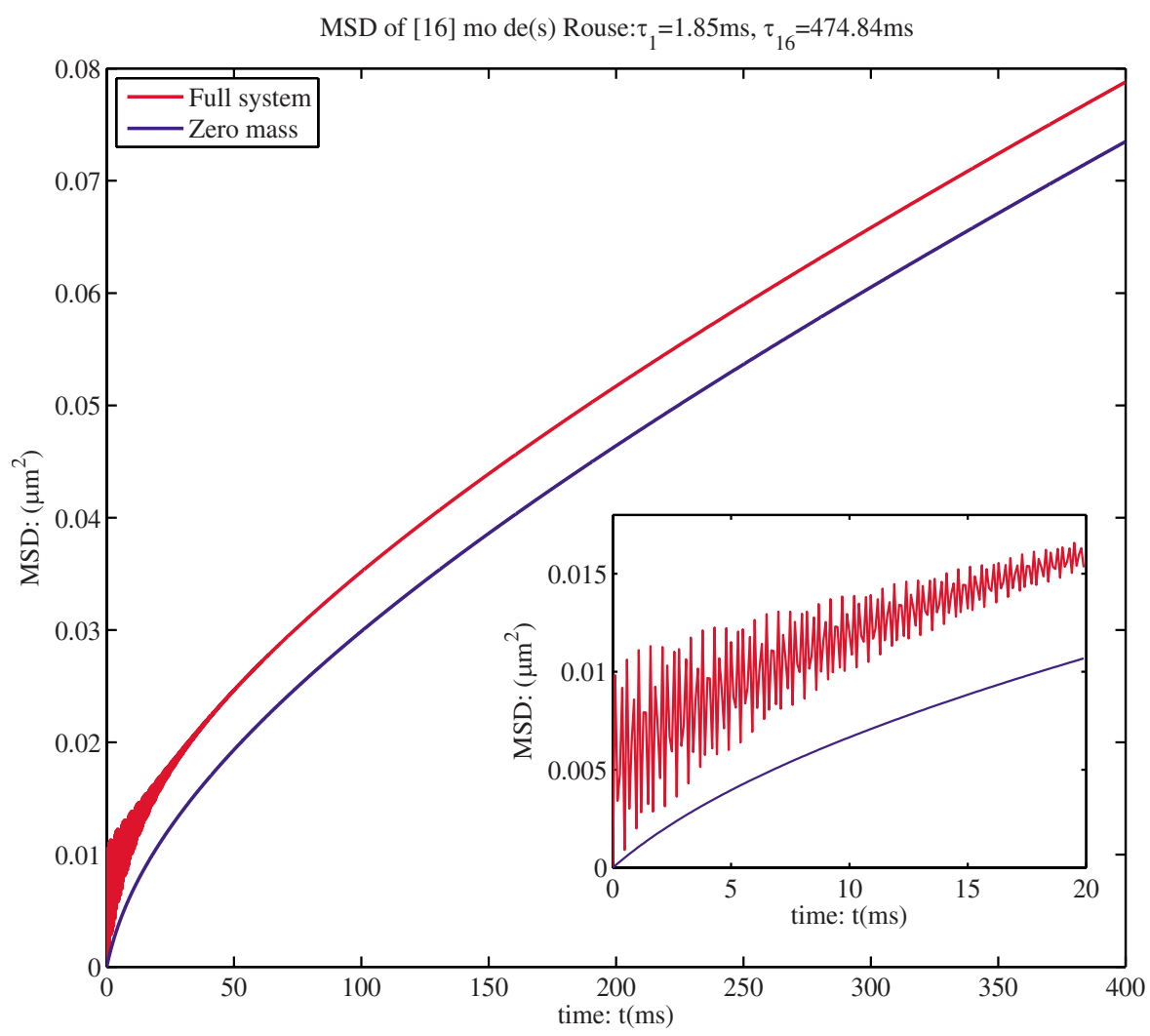

FIG. 3. MSD plotted for a GLE with a 16 -mode Rouse kernel $\left(\tau_{1}=1.85 \mathrm{~ms}\right.$ and $\left.\tau_{16}=474 \mathrm{~ms}\right)$, bead diameter of $1 \mu \mathrm{m}$, and mass $4.9 \times 10^{-9} \mathrm{mg}$. The upper curve is the MSD of the full GLE system; the lower curve is from the zero mass process. In the inset, we have magnified the short time scale dynamics, which feature oscillations due to inertia.

and identically distributed as a common universal random variable $c$, constrained only to have a finite fourth moment. For example, $c$ can be a standard normal or a chi-squared random variable.

This observation should prove useful when trying to conduct inference from experimental data. Rather than seeking to simultaneously optimize fitting to both the time scale spectrum and their relative weights, we may assume uniform weighting and apply inference methods on only three parameters: the shortest diffusive time $\tau_{1}$, the longest diffusive time $\tau_{N}$, and the scaling exponent, $\rho$. Alternatively, one can use the number $N$ of discrete spectra instead of the longest relaxation time. The weights can then be randomly perturbed without perturbing the MSD scaling behavior.

\section{E. Singular correction to the weak zero mass limit}

While the zero mass limit of the GLE (1) captures the desired transient MSD scaling behavior, numerical evaluation (see Fig. 3) of these profiles reveals two qualitative issues: first, when the mass is nonzero, we expect short-time oscillations due to inertia; second, there is a noticeable gap (vertical translation of the MSD curve) which does not close as the mass goes to zero. These two issues are indeed related. 
We address both of these issues in Sec. I B where we analyze solutions to the nonzero mass GLE as a singular perturbation of Eq. (14). The major result of this analysis is the exact characterization of a highly oscillatory correction process $Y(t)$ which entirely accounts for the finite-mass MSD gap in Fig. 3.

We find that we can write the $m>0$ solution $X_{m}(t)$ as a singular perturbation of the weak zero mass limit $X(t)$ derived in the preceding section [Eq. (14)]

$$
X_{m}(t)=X(t)+\sigma \sqrt{\frac{\bar{\alpha}}{2 N}} Y_{m}(t)+o(\sqrt{m}),
$$

where the stationary Gaussian correction term $Y$ is given by

$$
Y_{m}(t)=\int_{0}^{t} e^{-(1 / 2) \bar{\alpha}(t-s)} \cos [\omega(t-s)] d B(s),
$$

where the frequency of the oscillations is $\omega=\sqrt{N / m}$.

As mentioned earlier, the correction term $Y_{m}$ goes to zero weakly with diminishing mass in the sense that for any smooth rapidly decaying test function $\varphi$,

$$
\lim _{m \rightarrow \infty}\left\langle\left(\int_{0}^{T} Y_{m}(t) \varphi(t) d t\right)^{2}\right\rangle=0 .
$$

However, $\lim _{m \rightarrow 0}\left\langle Y_{m}(t)^{2}\right\rangle=1$. We take up this mathematical issue in a separate paper.

\section{CONCLUSION}

In this paper, we have presented an explicit family of GLEs together with closed-form solutions of the zero mass limit. These models generalize the classical Rouse model in terms of the scaling properties of the diffusive memory time scale spectrum. We then show the parametrized memory spectrum translates directly to control over the transient sub-diffusive MSD power law exponent and the time window of sub-diffusion, spanning the full range of sub-diffusive scaling. These results thereby establish a robust family of transient sub-diffusive models, for which the inverse problem of parameter inference from experimental data [cf. Fricks et al. (2009)] remains to be developed.

\section{ACKNOWLEDGMENTS}

The authors express appreciation to the community of scholars in the Virtual Lung Project at the University of North Carolina, where the subject of viscoelastic diffusion was introduced to us. In particular, we thank Jeremy Cribb, David Hill, Michael Rubinstein, and Rich Superfine for insights and numerous discussions. This paper is an outgrowth of collaborations with John Fricks and Tim Elston, whose input for this work remains invaluable. We also acknowledge insightful conversations with Joe DeSimone and Justin Hanes. We acknowledge important conversations with the probability community and we especially thank Peter March, Jonathan Mattingly, and Davar Khoshnevisan for their insights and feedback. Finally, we thank the anonymous referees for their highly constructive suggestions. The research of Forest and Yao have been supported in part by NIH Grant No. R01 HL077546-01A2 and NSF DMS Grants No. 0604891 and No. 0540779, while the research of McKinley has been supported in part by NSF DMS Grant No. 0449910. 


\section{APPENDIX A: DETAILS OF THE ZERO MASS LIMIT}

\section{The weak limit}

In what follows, we denote the unilateral Laplace transform of a given function $f(t)$ by

$$
\mathcal{L}\{f\}(z)=\widetilde{f}(z):=\int_{0}^{\infty} e^{-z t} f(t) d t .
$$

As noted in Adelman (1976) and Santamaria-Holek (2008), one can readily solve Eq. (1) for the transform of the position

$$
\tilde{X}(z)=\frac{z^{-1} \widetilde{F}(z)}{m z+\widetilde{\Gamma}(z)} .
$$

The inverse transform $\chi(t):=\mathcal{L}^{-1}\left\{[m z+\widetilde{\Gamma}(z)]^{-1}\right\}$ has been called the susceptibility [Adelman (1976)] and denoting the new noise $G(t):=\mathcal{L}^{-1}\left\{z^{-1} \widetilde{F}(z)\right\}$, one may write the real-time solution for the position as

$$
X(t)=\int_{0}^{t} \chi(t-s) G(s) d s,
$$

where we have assumed vanishing initial position and velocity. The Prony series assumption on the memory kernel permits a direct computation of the inverse Laplace transform in the zero mass limit. Note that

$$
\frac{1}{\beta} \widetilde{\Gamma}(z)=\sum_{k=1}^{N} \frac{1}{z+\alpha_{k}}=\frac{p^{\prime}(z)}{p(z)}
$$

where the polynomial $p$ is given in Eq. (18). The right-hand side of Eq. (A2) may be written as the product of a rational function and a function with no singularities. Therefore we may invert back into real variables using a partial fraction decomposition.

To this end, we decompose $F(t)$ into a sum of independent OU processes $\left\{F_{k}(t)\right\}_{k=1}^{N-1}$, each corresponding to one of the diffusive time scales in the Prony series. It follows from the autocorrelation function

$$
\left\langle F_{k}(t) F_{k}(s)\right\rangle=k_{B} T \beta e^{-\alpha_{k}|t-s|}
$$

that each $F_{k}(t)$ has the form

$$
F_{k}(t)=\sqrt{2 k_{B} T \beta \alpha_{k}} \int_{0}^{t} e^{-\alpha_{k}(t-s)} d W_{k}(s),
$$

where the $W_{k}(t)$ are independent standard Brownian motions. Therefore

$$
z^{-1} \widetilde{F}(z)=\sqrt{2 k_{B} T \beta} \sum_{k=1}^{N} \frac{\sqrt{\alpha_{k}}}{z+\alpha_{k}} \widetilde{W}_{k}(z) .
$$

Denoting $\sigma^{2}=2 k_{B} T \beta^{-1}$, we have

$$
\tilde{X}(z)=\sigma \sum_{k=1}^{N} \frac{p(z)}{p^{\prime}(z)\left(z+\alpha_{k}\right)} \sqrt{\alpha_{k}} \tilde{W}_{k}(z) .
$$

The critical observation is that $p(z) / p^{\prime}(z)\left(z+\alpha_{k}\right)$ is a rational function with whose numerator and denominator have the same degree. Furthermore all of its singularities are 
simple poles at the zeros of $p^{\prime}(z)$, which we denote $\left\{-r_{j}\right\}_{j=1}^{N-1}$. We have the following partial fraction decomposition

$$
\frac{p(z)}{p^{\prime}(z)\left(z+\alpha_{k}\right)}=\frac{1}{N}+\sum_{j=1}^{N-1} \frac{p\left(-r_{j}\right)}{p^{\prime \prime}\left(-r_{j}\right)\left(-r_{j}+\alpha_{k}\right)} \frac{1}{z+r_{j}} .
$$

On the Laplace side, integration by parts is the algebraic identity

$$
\frac{1}{z+r_{j}}=\frac{1}{r_{j}}\left(1-\frac{z}{z+r_{j}}\right)
$$

Substituting this into expression for $\widetilde{X}$ yields

$$
\tilde{X}(z)=\sigma\left(S_{1}-S_{2}\right)
$$

where

$$
\begin{gathered}
S_{1}:=\sum_{k=1}^{N}\left(\frac{1}{N}+\sum_{j=1}^{N-1} \frac{p\left(-r_{j}\right)}{p^{\prime \prime}\left(-r_{j}\right)\left(\alpha_{k}-r_{j}\right)} \frac{1}{r_{j}}\right) \sqrt{\alpha_{k}} \tilde{W}_{k}(z), \\
S_{2}:=\sum_{k=1}^{N} \sum_{j=1}^{N-1} \frac{p\left(-r_{j}\right)}{p^{\prime \prime}\left(-r_{j}\right)\left(\alpha_{k}-r_{j}\right) r_{j}} \frac{z}{z+r_{j}} \sqrt{\alpha_{k}} \tilde{W}(z) .
\end{gathered}
$$

After noting Eq. (A9) and then Eq. (A4), $S_{1}$ simplifies as follows:

$$
S_{1}=\frac{p(0)}{p^{\prime}(0)} \sum_{k=1}^{N} \frac{1}{\sqrt{\alpha_{k}}} \tilde{W}_{k}(z)=\frac{1}{\sqrt{N \bar{\tau}}} \widetilde{B}_{0}(z),
$$

where $B_{0}(t)$ is a standard Brownian motion given in law by the relationship

$$
\sum_{k=1}^{N} \frac{1}{\sqrt{\alpha_{k}}} W_{k}(t)=\left(\sum_{k=1}^{N} \frac{1}{\alpha_{k}}\right)^{1 / 2} B_{0}(t) .
$$

The second term, $S_{2}$, simplifies after reversing the order of summation

$$
S_{2}=\sigma \sum_{j=1}^{N-1} \frac{p\left(-r_{j}\right)}{p^{\prime \prime}\left(-r_{j}\right) r_{j}} \frac{z}{z+r_{j}} \widetilde{\xi}_{j}(z),
$$

where $\xi_{j}(t)=\sum_{k=1}^{N}\left[\sqrt{\alpha_{k}} /\left(\alpha_{k}-r_{j}\right)\right] W_{k}(t)$. As such, $\xi_{j}$ is a Brownian motion with variance parameter

$$
\sigma_{j}^{2}=\sum_{k=1}^{N} \frac{\alpha_{k}}{\left(\alpha_{k}-r_{j}\right)^{2}} .
$$

We now observe that these noises are independent. Indeed

$$
\left\langle\xi_{i}(t) \xi_{j}(s)\right\rangle=(t \wedge s) \sum_{k=1}^{N} \frac{\alpha_{k}}{\left(\alpha_{k}-r_{i}\right)\left(\alpha_{k}-r_{j}\right)},
$$

where $t \wedge s$ denotes the minimum of $t$ and $s$. In the case $i \neq j$, the sum can be rewritten in terms of the original polynomial $p$ from Eq. (18) 


$$
\left\langle\xi_{i}(t) \xi_{j}(s)\right\rangle=\frac{t \wedge s}{r_{i}-r_{j}}\left(r_{j} p^{\prime}\left(-r_{i}\right)-r_{i} p^{\prime}\left(-r_{j}\right)\right)=0,
$$

since the $\left\{-r_{j}\right\}$ are roots of $p^{\prime}$. The above implies that the $\left\{\xi_{j}\right\}$ are uncorrelated. Being jointly Gaussian, they are in fact independent. This applies even to the process $B_{0}$ since it can be viewed as $\xi_{0}$ adopting the convention that $r_{0}=0$.

After performing the inverse Laplace transform on Eq. (A11), we recover the constants and OU processes proposed in Eq. (15).

\section{Inertial diffusion as a singular perturbation}

To derive the exact solution (26) to the nonzero mass GLE, we begin by recalling the Laplace transform of the position process, this time without sending the mass to zero,

$$
\tilde{X}_{m}(z)=\sigma \sum_{k=1}^{N} \frac{p(z) \sqrt{\alpha_{k}}}{\left(m z p(z)+p^{\prime}(z)\right)\left(z+\alpha_{k}\right)} \tilde{W}_{k}(z) .
$$

As before, we must find the zeros of the denominator in order to invert the transform. To this end, we denote

$$
q(z)=m z p(z)+p^{\prime}(z) .
$$

For sufficiently small $m$, there are $N-1$ distinct, real roots for $q$, which are perturbed from the roots of $p^{\prime}$,

$$
-R_{j}=-r_{j}+o(m), \quad j=1,2, \ldots, N-1 .
$$

In this sense, the Laplace zero mass limit is the leading order approximation. We claim that the remaining two roots are complex: $-R$ and its conjugate $-R^{*}$, where

$$
-R=A+\frac{1}{\sqrt{m}} B i+o(\sqrt{m}) .
$$

Indeed, the polynomial $q(z)$ can be written

$$
q(z)=m z^{N+1}+m a z^{N}+m a_{2} z^{N-1}+\cdots+a_{N+1}+N z^{N-1}+b z^{N-2}+\cdots+b_{N-1},
$$

where the highest order coefficients are

$$
a=\sum_{k=1}^{N} \alpha_{k}, \quad b=(N-1) a .
$$

We substitute into Eq. (A24) and balancing the highest order terms yields the identities

$$
\begin{array}{ll}
\text { Order } m^{-1 / 2(N-1)}: & 0=B^{N+1}-N B^{N-1}, \\
\text { Order } m^{-1 / 2(N-2)}: & 0=2 N A-a N+b .
\end{array}
$$

We conclude

$$
-R=-\frac{1}{2 N}\left(\sum_{k=1}^{N} \alpha_{k}\right)-i \sqrt{\frac{N}{m}}+o(\sqrt{m}) .
$$

Turning our attention to Eq. (A20), similar to the evaluation of Eq. (A11) in the previous section, there is a partial fractions decomposition for each summand. Denote $p_{k}(z)$ $=p(z) /\left(z+\alpha_{k}\right)$. Then, 


$$
\frac{p_{k}(z)}{q(z)}=\frac{p_{k}(-R)}{q^{\prime}(-R)(z+R)}+\frac{p_{k}\left(-R^{*}\right)}{q^{\prime}\left(-R^{*}\right)\left(z+R^{*}\right)}+\sum_{j=1}^{N-1} \frac{p_{k}\left(-R_{j}\right)}{q^{\prime}\left(-R_{j}\right)\left(z+R_{j}\right)} .
$$

After integrating by parts algebraically, we have

$$
\frac{p_{k}(z)}{q(z)}=J_{1}+J_{2} z
$$

where

$$
\begin{gathered}
J_{k, 1}:=\frac{p_{k}(-R)}{q^{\prime}(-R) R}+\frac{p_{k}\left(-R^{*}\right)}{q^{\prime}\left(-R^{*}\right) R^{*}}+\sum_{j=1}^{N-1} \frac{p_{k}\left(-R_{j}\right)}{q^{\prime}\left(-R_{j}\right)} \frac{1}{R_{j}}, \\
J_{k, 2}:=\frac{p_{k}(-R)}{q^{\prime}(-R)(z+R) R}+\frac{p_{k}\left(-R^{*}\right)}{q^{\prime}\left(-R^{*}\right)\left(z+R^{*}\right) R^{*}}+\sum_{j=1}^{N-1} \frac{p_{k}\left(-R_{j}\right)}{p^{\prime \prime}\left(-R_{j}\right)\left(z+R_{j}\right) R_{j}} .
\end{gathered}
$$

Noting Eq. (A4) from the preceding section, we see that

$$
J_{k, 1}=\frac{p_{k}(0)}{q(0)}=\frac{p_{k}(0)}{p^{\prime}(0)}=\alpha_{k}^{-1} \text {. }
$$

As in the preceding section, the Laplace inverse transform of these terms contribute to a standard Brownian motion with diffusion coefficient $\sigma / \sqrt{N \bar{\tau}}$.

We turn our attention to $J_{k, 2}$. A quick calculation reveals the terms associated with the real roots of $q$ can be viewed as being perturbed from similar terms in the weak zero mass limit

$$
\frac{p_{k}\left(-R_{j}\right)}{q^{\prime}\left(-R_{j}\right)\left(z+R_{j}\right)}=\frac{p_{k}\left(-r_{j}\right)}{p^{\prime \prime}\left(-r_{j}\right)\left(z+r_{j}\right)}+o(\sqrt{m}) .
$$

In light of the observation that $q(0)=p^{\prime}(0)$ and comparing to Eq. (A9), we see that for each $k$, the complex terms in $J_{k, 1}$ must satisfy

$$
\frac{p_{k}(-R)}{q^{\prime}(-R) R}+\frac{p_{k}\left(-R^{*}\right)}{q^{\prime}\left(-R^{*}\right) R^{*}}=\frac{1}{N}+o(\sqrt{m})
$$

which is to say

$$
\mathfrak{R e}\left(\frac{p_{k}(-R)}{q^{\prime}(-R) R}\right)=\frac{1}{2 N}+o(\sqrt{m}) .
$$

A more detailed calculation is required to discover that

$$
\mathfrak{I m}\left(\frac{p_{k}(-R)}{q^{\prime}(-R) R}\right)=o(\sqrt{m}) .
$$

To this end, we recall that $-R=-\bar{\alpha} / 2+i \sqrt{N / m}+o(\sqrt{m})$ and expand the numerator and denominator in terms of powers of $m^{-1 / 2}$,

$$
p_{k}(-R)=\Pi_{j \neq k}\left(\alpha_{j}-R\right)=(i \sqrt{N / M})^{N-1}(1+o(\sqrt{m}+i \sqrt{m})),
$$

while, in light of Eq. (A24), we have

$$
q^{\prime}(z)=m(N+1) z^{N}+N(N-1) z^{N-2}
$$

so that 


$$
q^{\prime}(-R) R=(i \sqrt{N / m})^{N-1}(-2 N+o(\sqrt{m}+i \sqrt{m})) .
$$

Together this implies Eq. (A35).

We may now complete the evaluation of $J_{k, 2}$. Writing $A_{k}:=p_{k}(-R) / q^{\prime}(-R) R, \omega$ $=\sqrt{N / m}$, and again recalling the form of $R$, Eq. (A27), we have the identity

$$
\frac{A_{k}}{z+R}+\frac{A_{k}^{*}}{z+R^{*}}=\mathfrak{R e}\left(A_{k}\right) \frac{z+c_{k}}{\left(z-\frac{1}{2} \bar{\alpha}\right)^{2}+\omega^{2}},
$$

where

$$
c_{k}=-\frac{1}{2} \bar{\alpha}-\omega \frac{\mathfrak{I m}\left(A_{k}\right)}{\mathfrak{R e}\left(A_{k}\right)} .
$$

The Laplace inverse transform of the associated term is

$$
\begin{aligned}
\mathcal{L}^{-1}\left[\frac{z+c_{k}}{\left(z-\frac{1}{2} \bar{\alpha}\right)^{2}+\omega^{2}} \mathfrak{R e}\left(A_{k}\right) \sqrt{\alpha_{k} z} \tilde{W}(z)\right] \\
=\operatorname{Re}\left(A_{k}\right) \sqrt{\alpha_{k}} \int_{0}^{t} e^{-(1 / 2) \bar{\alpha}(t-s)}\left[\cos (\omega(t-s))+\left(\frac{c_{k}+\frac{1}{2} \bar{\alpha}}{\omega}\right) \sin (\omega(t-s))\right] d W_{k}(s) \\
=\operatorname{Re}\left(A_{k}\right) \sqrt{\alpha_{k}} \int_{0}^{t} e^{-(1 / 2) \bar{\alpha}(t-s)}\left[\cos (\omega(t-s))+\left(\frac{\mathfrak{I m}\left(A_{k}\right)}{\mathfrak{R e}\left(A_{k}\right)}\right) \sin (\omega(t-s))\right] d W_{k}(s) .
\end{aligned}
$$

Noting the order of the coefficient of the sine term $\sqrt{\alpha_{k}} \mathfrak{I m}\left(A_{k}\right)=o(\sqrt{m})$, we conclude that $X_{m}(t)$ has an order 1 term of the form

$$
Y_{m}(t)=\sum_{k=1}^{N} J_{k, 2}=\sqrt{\frac{\bar{\alpha}}{2 N}} \int_{0}^{t} e^{-(1 / 2) \bar{\alpha}(t-s)} \cos [\omega(t-s)] d B_{y}(s) .
$$

This is precisely the process $Y$ appearing in Eq. (27).

\section{APPENDIX B: ROBUSTNESS OF THE ANOMALOUS EXPONENT}

As mentioned, the model is robust in that for random coefficients the anomalous scaling is still seen. We demonstrate this by showing a pointwise in $t$ convergence for the given family of $c_{k, N}$. In fact, a stronger result can be given, but we defer this finer analysis to another venue.

Intuitively, we can think of this probabilistic result as follows. For a given $\rho$, there is mean function $\mu(t)$ defined below that corresponds to the MSD when the coefficients are chosen to be exactly uniform. If we perturb these coefficients randomly, the larger that $N$ is, the smaller the effect of the perturbation will be. In light of this observation, when performing statistical inference on actual data, one can allow for some uncertainty in the coefficients while seeking a precise estimate for $\rho$. As long as there is no systematic structure in the coefficients, the following characterization of intermediate time scale holds. 
Let a random family of independent and identically distributed coefficients $\left\{c_{k, N}\right\}_{k \leq N}$ be fixed. As shown in Sec. II D, the contribution of the Brownian term can be ignored when establishing the intermediate dynamics. Therefore letting $c_{0}=0$ for simplicity, the MSD of each $x_{N}(t)$ is given by

$$
\left\langle x_{N}^{2}(t)\right\rangle=\frac{1}{N} \sum_{k=1}^{N} \frac{c_{k}^{2}}{\alpha_{k}}\left(1-e^{-\alpha_{k} t}\right) .
$$

Fixing $t$, we wish to show convergence of the sequence of points $\left\{\left\langle x_{N}(t)\right\rangle\right\}_{N=1}^{\infty}$ to a function that is proportional to the value

$$
\mu(t):=\lim _{N \rightarrow \infty} \mu_{N}(t),
$$

where the functions $\mu_{N}$ were defined above. To do this, we now look at the distribution of $\left\langle x_{N}^{2}(t)\right\rangle$ with respect to the probability measure $\mathbb{P}$ used to select the family of coefficients. If we use $\mathbb{E}$ and var to denote the average and variance with respect to $\mathbb{P}$, it suffices to show that

$$
\lim _{N \rightarrow \infty} \operatorname{var}\left(\left\langle x_{N}^{2}(t)\right\rangle\right)=0
$$

Indeed, denoting $\sigma_{c}^{2}=\mathbb{E}\left[c_{k}^{2}\right]$,

$$
\operatorname{var}\left(\left\langle x_{N}^{2}(t)\right\rangle\right)=\mathbb{E}\left[\left(\frac{1}{N} \sum_{k=1}^{N} \frac{c_{k}^{2}-\sigma_{c}^{2}}{\alpha_{k}}\left(1-e^{-\alpha_{k} t}\right)\right)^{2}\right]=\frac{1}{N^{2}} \sum_{k=1}^{N} \operatorname{var}\left(c_{k}^{2}\right)\left(\frac{1-e^{-\alpha_{k} t}}{\alpha_{k}}\right)^{2} .
$$

No cross terms appear because $\mathbb{E}\left[\left(c_{k}^{2}-\sigma_{c}^{2}\right)\left(c_{j}^{2}-\sigma_{c}^{2}\right)\right]=0$ due to the independence of the $c_{k}$.

It remains to recognize a Riemann approximation again, namely,

$$
J:=\sum_{k=1}^{N}\left(\frac{1-e^{-\alpha_{k} t}}{\alpha_{k}}\right)^{2} \frac{1}{N}=\tau_{1}^{2} \int_{0}^{1} \frac{1}{x^{2 p}}\left(1-e^{-x^{p} t / \tau_{1}}\right)^{2} d x+o\left(\frac{1}{N}\right)
$$

is a convergent definite integral. As such

$$
\operatorname{var}\left(\left\langle x_{N}^{2}(t)\right\rangle\right)=\frac{1}{N} \operatorname{var}\left(c^{2}\right) J+o\left(\frac{1}{N}\right),
$$

which tends to zero with $N$.

\section{References}

Adelman, S. A., "Fokker-Planck equations for simple non-Markovian systems," J. Chem. Phys. 64, 124-130 (1976).

Doi, M., and S. F. Edwards, The Theory of Polymer Physics (Oxford University Press, New York, 1986).

Fricks, J., L. Yao, T. C. Elston, and M. G. Forest, "Time-domain methods for diffusive transport in soft matter," SIAM J. Appl. Math. 69(5), 1277-1308 (2009).

Gratton, S. E. A., P. A. Ropp, P. D. Pohlhaus, J. C. Luft, V. J. Madden, M. E. Napier, and J. M. DeSimone, “The effect of particle design on cellular internalization pathways," Proc. Natl. Acad. Sci. U.S.A. 105(33), 11613-11618 (2008).

Kou, S. C., and X. S. Xie, "Generalized Langevin equation with fractional Gaussian noise: Subdiffusion with in a single protein molecule," Phys. Rev. Lett. 93(18), 180603 (2004).

Kremer, K., and G. S. Grest, "Dynamics of entangled linear polymer melts: A molecular-dynamics simulation," 
J. Chem. Phys. 92(8), 5057-5086 (1990).

Kubo, R., Statistical Physics II: Nonequilibrium Statistical Mechanics, 2nd ed. (Springer, New York, 1985).

Lai, S. K., D. E. O’Hanlon, S. Harrold, S. T. Man, Y.-Y. Wang, R. Cone, and J. Hanes, "Rapid transport of large polymeric nanoparticles in fresh undiluted human mucus," Proc. Natl. Acad. Sci. U.S.A. 104(5), 1482-1487 (2007).

Lai, S. K., Y.-Y. Wang, R. Cone, D. Wirtz, and J. Hanes, “Altering mucus rheology to 'solidify' human mucus at the nanoscale," PLoS ONE 4(1), e4294 (2009).

Levine, A. J., and T. C. Lubensky, "Response function of a sphere in a viscoelastic two-fluid medium," Phys. Rev. E 63(4), 041510 (2001).

Mason, T. G., and D. A. Weitz, "Optical measurements of frequency-dependent linear viscoelastic moduli of complex fluids,” Phys. Rev. Lett. 74(7), 1250-1253 (1995).

Matsui, H., V. E. Wagner, D. B. Hill, U. E. Schwab, T. D. Rogers, B. Button II, R. M. Taylor II, R. Superfine, M. Rubinstein, B. H. Iglewski, and R. Boucher, "A physical linkage between cystic fibrosis airway surface dehydration and pseudomonas aeruginosa biofilms," Proc. Natl. Acad. Sci. U.S.A. 103(48), 18131-18136 (2006).

Morgado, R., F. A. Oliveira, G. G. Batrouni, and A. Hansen, "Relation between anomalous diffusion and normal diffusion in systems with memory," Phys. Rev. Lett. 89(10), 100601 (2002).

Mori, H., "Transport, Collective Motion, and Brownian Motion," Prog. Theor. Phys. 33, 423-455 (1965).

Rouse, P. E., "A theory of the linear viscoelastic properties of solutions of coiling polymers," J. Chem. Phys. 21(12), 1272-1280 (1953).

Rubinstein, M., and R. H. Colby, Polymer Physics (Oxford University Press, New York, 2003).

Santamaria-Holek, I., "Anomalous diffusion in microrheology: A comparative study," AIP Conf. Proc. 982(1), 672-677 (2008).

Suh, J., M. Dawson, and J. Hanes, "Real-time multiple-particle tracking: applications to drug and gene delivery," Adv. Drug Delivery Rev. 57, 63-78 (2005).

Suk, J. S., S. K. Lai, Y.-Y. Wang, L. M. Ensign, P. L. Zeitlin, M. P. Boyle, and J. Hanes, "The penetration of fresh undiluted sputum expectorated by cystic fibrosis patients by non-adhesive polymer nanoparticles," Biomaterials 30(13), 2591-2597 (2009).

Vainstein, M. H., L. C. Lapas, and F. Oliviera, “Anomalous diffusion,” Acta Phys. Pol. B 39(5), 1273-1281 (2008).

Zimm, B. H., "Dynamics of polymer molecules in dilute solution: viscoelasticity, flow birefringence and dielectric loss," J. Chem. Phys. 24(2), 269-278 (1956).

Zwanzig, R., and M. Bixon, "Hydrodynamic theory of the velocity correlation function," Phys. Rev. A 2(5), 2005-2012 (1970). 